\title{
Hemorrhagic Bullous Cellulitis/Necrotizing Fasciitis of the Foot due to $V$. fluvialis and Complicated by E. cloacae Osteomyelitis
}

\author{
Seyedqumar Mirfendereski, Naveen Pathak, Tejas Patel, Kelly L. Cervellione, Sameer Sharma and \\ Farshad Bagheri*
}

Departments of Internal Medicine and Clinical Research, Jamaica Hospital Medical Center, Jamaica, NY 11418, USA

\begin{abstract}
Vibrio fluvialis (V. fluvialis) is a halophilic bacterium that is most often associated with gastrointestinal tract infections after consumption of contaminated seafood. The predominant presenting symptoms of $V$. Fluvialis infection include explosive diarrhea, vomiting, abdominal pain and dehydration. Vibrio species, V. Fluvialis in particular, are rarely a cause of necrotizing fasciitis. We describe a case of a 56-year-old male presenting with complaints of redness and swelling of and discharge from his right toes. The patient was diagnosed with necrotizing fasciitis and septicemia. Debridement of the areas proved unsuccessful; amputation of infected phalanges was effective in preventing spread of disease. Cultures were positive for $V$. fluvialis from the bullae aspirates and Enterobacter cloacae from the bone culture. The patient recovered successfully from both infections. This case is atypical because of the absence of any gastrointestinal symptoms and the presence of necrotizing fasciitis and septicemia due to V. Fluvialis infection.
\end{abstract}

\section{INTRODUCTION}

Vibrio fluvialis (V. fluvialis), a member of the Vibrio family, is a halophilic, gram negative, motile bacterium that is most often associated with gastrointestinal tract infections after consumption of contaminated seafood. V. fluvialis are naturally found in marine, estuarine, and brackish waters around the world, including the United States. The presenting symptoms of patients who have acquired $V$. fluvialis most frequently include explosive diarrhea, vomiting, abdominal pain, and dehydration [1, 2] and can often mimic food poisoning. Other, more rarely reported manifestations of infection due to $V$. fluvialis include wound infections [35], otitis [6] and septicemia [7]. Necrotizing fasciitis (NF), commonly known as the flesh-eating disease, is a severe bacterial infection that causes soft tissue to decay rapidly. Recent reports suggest that the pathogens most often associated with development of NF are staphylococcus aureus, particularly methicillin-resistant strains $[8,9]$; however there have been numerous case reports describing NF caused by a vast variety of micro-organisms. While numerous reports have linked Vibrio vulnificus to development of NF, V. fluvialis has only been reported as causing NF in a handful of patients [10]. We report a case of a middle-aged male who, after exposure to sea water, developed hemorrhagic bullous cellulitis/necrotizing fasciitis that was further complicated by deeper subcutaneous involvement and osteomyelitis with Enterobacter cloacae (E. cloacae) co-infection. In addition, he had clinically significant septicemia. These infections resulted in amputation of three affected toes. This case is a typical because necrotizing fasciitis was the presenting

*Address correspondence to this author at the Departments of Internal Medicine and Clinical Research, Jamaica Hospital Medical Center, Jamaica, NY 11418, USA; E-mail: fbagheri@jhmc.org symptom of $V$. fluvialis infection, as well as the co-occurring development of E. cloacae osteomyelitis and the presence of septicemia.

\section{CASE REPORT}

A 56-year-old Hispanic male presented to the emergency department complaining of redness and swelling of his right toes that began after visiting a beach in Jamaica one week prior to the current admission. In addition, he began experiencing discharge from his fourth right toe which started three days prior to admission. Two days before he presented, he was given oral antibiotics (cefaclor $750 \mathrm{mg}$ BID) by his primary care physician with no improvement in symptoms. The patient did not recall any trauma or abrasion to his right foot. Prior to this illness the patient was active and independent. He had a history of type 2 diabetes mellitus, diabetic peripheral neuropathy, chronic renal failure and coronary artery disease. He had no history of liver disease or alcoholism. He denied eating any fresh oysters or seafood prior to the onset of the current illness.

On admission to the emergency department the patient was febrile and was complaining of hiccups for the last five days. He was alert, oriented, and normotensive. On physical examination the right foot was swollen erythematous with the fourth toe more swollen; there was accompanying hemorrhagic bullae with foul smelling drainage. Initial treatment for cellulitis included intravenous fluids and ampicillin/sulbactam 3mg IVPB Q6h. Hematology and biochemistry results revealed leukocytosis, with a white blood cell count (WBC) of 22.7. Other labs of interest included neutrophils of $75 \%$ and bands of 9, red blood cell count (RBC) of 3.07, hemoglobin (HGB) of 9.5, hematocrit (HCT) of 28.2 and Creactive protein $(\mathrm{CRP})$ of $9.0 \mathrm{mg} / \mathrm{dl}$. X-ray of the right foot 
showed gas within the soft tissues of the fourth and fifth toes (Fig. 1). MRI of same area revealed mild marrow edema in second, third and fourth proximal phalanges (Fig. 2). On the second day of admission, the patient underwent debridement. Due to the findings on the $\mathrm{x}$-ray, halophilic Vibrio was suspected. Therefore, the antibiotic regimen was reviewed and modified to include doxycyclin 100mg IVBP BID, imipenem 500mg IVBP Q6h, and clindamycin 600 mg IVBP Q8h.

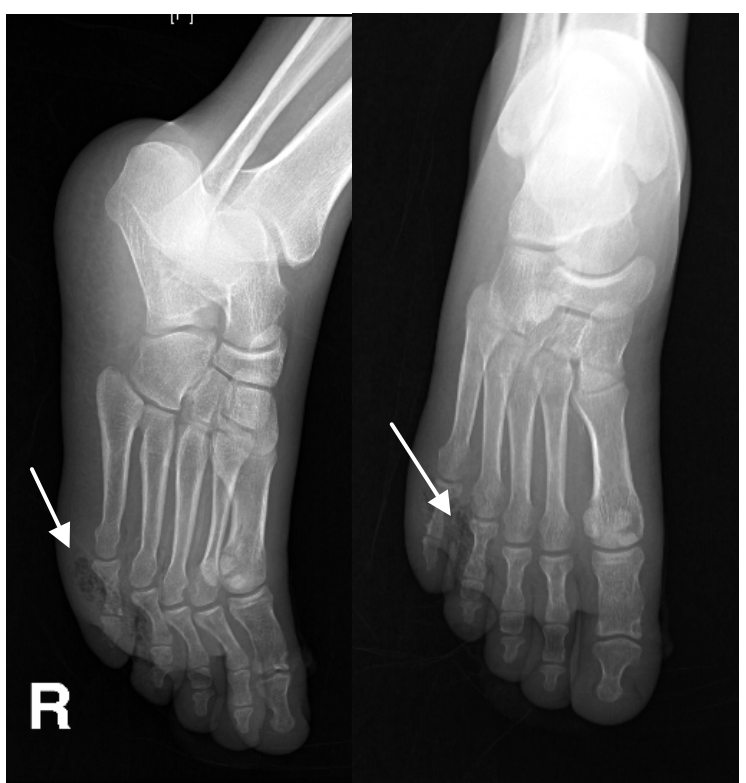

Fig. (1). X-ray showing subcutaneous gas overlying the right distal lateral foot

Two wound cultures were sent from bullae aspirates for Vibrio species. Due to the gas forming infection, necrotizing cellulitis, and the presence of clinically significant septicemia, the patient underwent amputation of the affected toes 5 days after admission. Post-surgery, results of the cultures were returned which showed $V$. fluvialis from the bullae aspirates and Enterobacter cloacae (E. cloacae) from the bone culture specimen. The patient was discharged to in-patient rehabilitation 5 days after amputation on intravenous antibiotics to complete treatment for osteomyelitis. At discharge, WBC count had improved to 13.0 (from 22.7) and CRP had improved to 1.8 (from 9.0); there were no significant changes in neutrophil counts, RBC, HGB or HCT.
At one month follow-up, the patient remained stable without reoccurrence of symptoms. The patient was lost to follow-up for 18 months, but recently had a follow-up x-ray of the right foot because of foot swelling. Three views showed soft tissue swelling with no soft tissue emphysema. The extensive destructive change in the mid-foot and subluxation at the second and third metatarsophalangeal joints was diagnosed as Charcot's joint (diabetic arthropathy). There has been no reoccurrence of symptoms related to $V$. fluvialis infection, E. cloacae osteomyelitis, septicemia, or necrotizing fasciitis during the 18 months.

\section{DISCUSSION}

\section{Pathogenesis and Presentation}

Due to the infrequent reporting of $V$. fluvialis, the epidemiology of the infection and the mechanisms of pathogenicity are not sufficiently understood $[11,12]$. With the rising number of reports of cases of this particular strain of Vibrio, it is expected that more information regarding pathogenesis will be available for physicians in the near future [11]. The bacterium could produce (i) a factor which causes $\mathrm{CHO}$ cell elongation $(\mathrm{CEF})$ similar to that elicited by V. cholerae enterotoxin and by the heat-labile enterotoxin of Escherichia coli, (ii) cytolysin(s) active against erythrocytes, (iii) nonhemolytic, CHO cell-killing factor(s), and (iv) protease(s) active against azocasein [1].

As previously noted, diarrhea, mild to moderate dehydration, vomiting and abdominal pain are the most common presentations of patients infected with V. Fluvialis [1]. For extraintestinal sites, clinical presentations can include wound infection and septicemias. Wound infections from Vibrio's occur after exposure of a break in skin to infected seawater, fish or shellfish. Wound infection may be mild and selflimited or, as in the case presented here, severe and invasive. Most often, wound infection caused by Vibrio species are from the alginolyticis, damsela, or vulnificus organisms; wound infection due to fluvialis, hollisae, and parahaemolyticus organisms are more rare [10]. Furthermore, septicemia caused by $V$. fluvialis infection is also very rare [10].

Vibrio species, $V$. fluvialis in particular, are rarely a cause of NF [10]. In a recent review of all cases of NF reported in a 16-month period in Taiwan, 13 of 52 were due to Vibrio

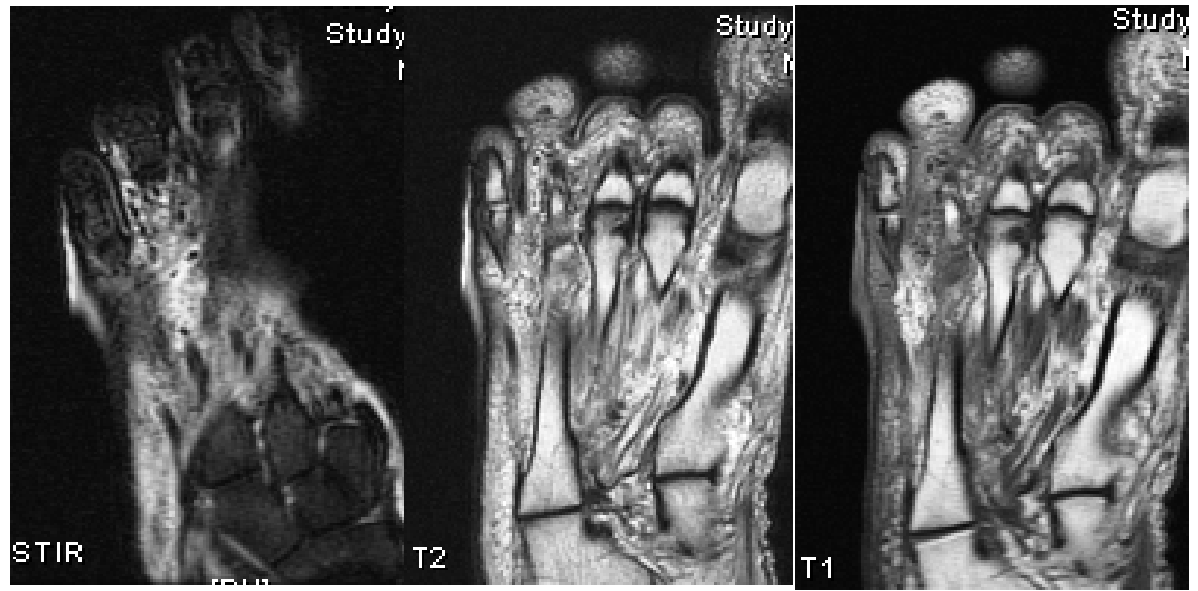

Fig. (2). MRI showing mild marrow edema within the second, fourth and fifth metatarsal, indicative of osteomyelitis 
infections, but only 1 of these were caused by $V$. fluvialis [10]. Findings from the Taiwan study suggested that diabetes mellitus $(\mathrm{N}=7)$ may predispose an individual to developing $\mathrm{NF}$ as a symptom of Vibrio infection; this finding has also been reported in other cases [13]. Since our patient had been previously diagnosed with Diabetes Mellitus Type 2, this case provides further support for the hypothesis that it may be a contributing factor to this type of wound infection presentation.

In addition to $V$. fluvialis, cultures also revealed the presence of E. cloacae in our case. E. cloacae infection often occurs in patients with extended hospital stays and in those who have pre-existing skin and soft tissue infections. The presence of the bacteria carries a considerable risk for the development of sepsis and, ultimately, mortality. The presence of the E. cloacae osteomyelitis in our case was most likely acquired post-Vibrio infection, yet may have influenced the progression of NF and sepsis development. We cannot be sure whether the septicemia in this case was caused by the $V$. fluvialis, E. cloacae infection, or a combination of the two. Since the diagnosis and treatment were made in a timely manner, further complications were avoided.

\section{Diagnosis and Treatment}

Most, if not all, strains of $V$. fluvialis are resistant to at least one antimicrobial $[14,15]$. In a study of drug resistance in 19 strains of $V$. fluvialis [14], all were found to be resistant to at least one antimicrobial; $75 \%$ of the strains were resistant to ampicillin, furazolidone, nalidixic acid, and streptomycin. Other antimicrobials detected on antibiogram in one or more strains included chloramphenicol (35\%), gentamicin $(40 \%)$, tetracycline $(10 \%)$, neomycin $(40 \%)$, sulfamethoxazole $(30 \%)$, sulfadiazine $(20 \%)$, trimethoprim $(15 \%)$ and ciprofloxacin $(5 \%)$. Therefore, recommended medical treatment for wound infection due to $V$. fluvialis is administration of at least two antimicrobial agents, including ceftazidime, cefotaxime, doxycycline, and/or ciprofloxacin [13].

Adding to the difficulties associated with treatment of $V$. fluvialis, commercially available tests for identification of Vibrio species have proven to have low specificities and often require modification of normal testing procedures (e.g. inoculation of isolate, absence of $\mathrm{NaCl}$, etc.) [16]. Without proper identification of species, treatment decisions are skewed and can be potentially fatal to patients.

Early detection and treatment of Vibrio infection in patients with hemorrhagic bullous cellulitis/necrotizing fascitis and sepsis is critical to the patient's survival. If Vibrio infection is suspected in cases of bullous cellulitis/necrotizing fascitis, treatment should begin as early as possible in order to prevent potentially deadly spread of the infection. Scientific literature recommends amputation early after detection of necrotizing fasciitis and Vibrio infection in order to prevent migration [13].

\section{Prognosis}

With the development of more effective treatment over the past few decades, the prognosis of patients acquiring
Vibrio infections has improved. Most cases that are gastroenterological in nature are often mild. High mortality rates are still seen, however, in patients who develop sepsis, have an underlying chronic liver disease, have an underlying immuno-compromising disease, or develop necrotizing fasciitis $[10,17,18]$. Our patient was an exception to the high mortality rates often seen in this kind of presentation, especially given the co-occurrence of E. cloacae osteomyelitis and clinically significant sepsis.

\section{CONCLUSIONS}

This is an interesting and important case to report due to the rarity of this type of presentation of $V$. fluvialis infection and the high priority for quick and accurate diagnosis and treatment in order to prevent potentially fatal spread of infection. If a patient presents with these types of symptoms and has recently had contact with seawater or raw or potentially undercooked seafood, Vibrio infection should be suspected and treated immediately.

\section{REFERENCES}

[1] Huq MI, Alam AK, Brenner DJ, et al. Isolation of Vibrio-like group, EF-6, from patients with diarrhea. J Clin Microbiol 1980; 11: 621-4.

[2] Allton DR, Forgione MA Jr, Gros SP. Cholera-like presentation in Vibrio fluvialis enteritis. South Med J 2006; 99: 765-7.

[3] Klontz KC, Desenclos JC. Clinical and epidemiological features of sporadic infections with Vibrio fluvialis in Florida, USA. J Diarrhoeal Dis Res 1990; 8: 24-6.

[4] Huang KC, Hsu RW. Vibrio fluvialis hemorrhagic cellulitis and cerebritis. Clin Infect Dis 2005; 40: e75-7.

[5] Varghese MR, Farr RW, Max MK, et al. Vibrio fluvialis wound infection associated with medicinal leech therapy. Clin Infect Dis 1996; 22: 709-10.

[6] Cabrera Rodriguez LE, Monrov SP, Morier L, et al. Severe otitis due to Vibrio fluvialis in a patient with AIDs: first report in the World. Rev Cubana Med Trop 2005; 57: 154-5.

[7] Levine WC, Griffin PM. Vibrio infections on the Gulf Coast: Results of first year of regional surveillance. Gulf Coast Vibrio Working Group. J Infect Dis 1993; 167: 479-83.

[8] Young LM, Price CS. Community-acquired methicillin-resistant Staphyloccocus aureas emerging as important cause of necrotizing fasciitis. Surg Infect (Larchmt) 2008; April 9 [e-pub ahead of print].

[9] Wijaya L, Hau LY, Kurup A. Community-associated methicillinresistant Staphylococcus aureus: overview and local situation. Ann Acad Med Singapore 2006; 35: 179-86.

[10] Tsai Y, Hsu RW, Huang K, et al. Systemic vibrio infection presenting as necrotizing fascitis and sepsis: a series of thirteen cases. J Bone Joint Surg Am 2004; 86A: 2497-502.

[11] Chakraborty R, Chakraborty S, De K, et al. Cytotoxic and cell vacuolating activity of Vibrio fluvialis isolated from paediatric patients with diarrhea. J Med Microbiol 2005; 54: 707-16.

[12] Janda JM, Powers C, Bryant RG, Abbott SL. Current perspectives on the epidemiology and pathogenesis of clinically significant $\mathrm{Vi}$ brio spp. Clin Microbiol Rev 1988; 1: 245-67.

[13] Daniels NA, Shafaie A. A review of pathogenic Vibrio infections for clinicians. Infect Med 2000; 17: 665-85.

[14] Srinivasan VB, Virk RK, Kaundal A, et al. Mechanism of drug resistance in clonally related clinical isolates of Vibrio fluvialis isolated in Kalkata, India. Antimicrob Agents Chemother 2006; 50: 2428-32.

[15] Ahmed AM, Nakagawa T, Arakawa E, Ramamurthy T, Shinoda S Shimamoto T. New aminoglycoside acetyltransferase gene, aac(3)$I d$, in a class 1 integron from a multiresistant strain of Vibrio fluvialis isolated from an infant aged 6 months. J Antimicrob Chemother 2004; 53: 947-51.

[16] O’Hara CM, Sowers EV, Bopp CA, Duda SB, Strockbine NA. Accuracy of six commercially available systems for identification of members of the family Vibrianaceae. J Clin Microbiol 2003; 41: 5654-9. 
[17] Morris JG. Cholera and other types of vibriosis: a story of human pandemics and oysters on the half shell. Clin Infect Dis 2003; 37: $272-80$.
[18] Wongpaitoon V, Sathapatayavongs B, Prachaktam R, Bunyaratvej S, Kurathong S. Spontanious Vibrio vulnificus peritonitis and primary sepsis in two patients with alcoholic cirrhosis. Am J Gastroenterol 1985; 80: 706-8.

(C) Mirfendereski et al.; Licensee Bentham Open.

This is an open access article licensed under the terms of the Creative Commons Attribution Non-Commercial License (http://creativecommons.org/licenses/ by-nc/3.0/) which permits unrestricted, non-commercial use, distribution and reproduction in any medium, provided the work is properly cited. 